\title{
The Challenges to Coupling Dynamic Geospatial Models
}

N. Goldstein

June 23, 2006

Geoscapes: A Journal of Geography and Geospatial Information and Collections 
This document was prepared as an account of work sponsored by an agency of the United States Government. Neither the United States Government nor the University of California nor any of their employees, makes any warranty, express or implied, or assumes any legal liability or responsibility for the accuracy, completeness, or usefulness of any information, apparatus, product, or process disclosed, or represents that its use would not infringe privately owned rights. Reference herein to any specific commercial product, process, or service by trade name, trademark, manufacturer, or otherwise, does not necessarily constitute or imply its endorsement, recommendation, or favoring by the United States Government or the University of California. The views and opinions of authors expressed herein do not necessarily state or reflect those of the United States Government or the University of California, and shall not be used for advertising or product endorsement purposes. 


\title{
The Challenges to Coupling Dynamic Geospatial Models
}

\author{
ABSTRACT \\ Many applications of modeling spatial dynamic systems focus on a single system and a single \\ process, ignoring the geographic and systemic context of the processes being modeled. A \\ solution to this problem is the coupled modeling of spatial dynamic systems. Coupled modeling \\ is challenging for both technical reasons, as well as conceptual reasons. This paper explores the \\ benefits and challenges to coupling or linking spatial dynamic models, from loose coupling, \\ where information transfer between models is done by hand, to tight coupling, where two (or \\ more) models are merged as one. To illustrate the challenges, a coupled model of Urbanization \\ and Wildfire Risk is presented. This model, called Vesta, was applied to the Santa Barbara, \\ California region (using real geospatial data), where Urbanization and Wildfires occur and recur, \\ respectively. The preliminary results of the model coupling illustrate that coupled modeling can \\ lead to insight into the consequences of processes acting on their own.
}

\author{
Noah Goldstein \\ Applied Statistics \& Economics Group \\ Systems \& Decision Sciences Section \\ Lawrence Livermore National Laboratory \\ 7000 East Ave., L-644 \\ Livermore, CA 94550-9234 \\ goldstein8@1lnl.gov \\ Tel: 935-423-3916 \\ Fax: 925-423-7914
}




\section{Introduction}

Dynamic Geospatial Models (DGM) is a broad term used to refer to computational models of a problem's or process' change over the earth in a specified period of time. Since their inception DGM's have been used in virtually all disciplines to express spatiotemporal behavior of entities from the micro to macro scale. In a geographic context, DGM's have had wide applications in the sciences, particularly those with environmental emphases.

There are a number of benefits and challenges to using and creating DGM's for both theoretical and applied purposes. The benefits to including a DGM in one's analytical or applied work is that the process or problem studied can readily be visualized either in a GIS or a graphical output. As computation and technology has improved, the quality and sophistication of the visual analysis has increased dramatically. A second benefit to the incorporation of a DGM is that it can be used as a forecasting tool for policy and scenario testing. Through various model parameterizations and seeding of DGM's, multiple realizations of environmental, social, and policy processes can be created. This in turn leads to yet another benefit of DGM's; through their forecasting ability and visualization tools, DGM's can add knowledge to the process of understanding an event or process by stakeholders and scientists alike.

However profound the benefits to using DGM's, their inherent challenges should not be overlooked. These include the formalization of the process to be modeled, understanding the results of the simulation, and the context of the modeling approach. A significant first step in using a DGM is in the formalization of the modeling problem itself. This is an issue at both ends of the theoretical- applied modeling spectrum. In theoretical modeling, one begins with theories and then creates a modeling framework that represents the simplest "universe" of that theory, ignoring the rich tapestry of the "real world". In applied modeling, one generalizes the 
infinitesimally complex and complicated components of the "real world" into a computer formalization.

A similarly significant problem with creating and using a DGM is in understanding and modeling the context of the problem. Usually models are created to analyze, forecast or visualize one system, and the "environment", or anything external to the model is simplified or ignored. The formalization process of the model necessitates the identification of the problem definition and boundaries. For many modeling exercises this may be an appropriate approach. Yet for many modeling contexts, the "single system" approach can be viewed as myopic, as the environment is not static and evolves or changes as well as the modeled process.

There are many situations where modeling both a single system and the environment (or another system) adds significant value. For example, a simple stream-flow model can tell the user what the impact of a large rainstorm can have on a watershed. All one needs for a simple model is the slope, soil type and stream fetch for the watershed, and some deterministic, physicsbased formulae on fluid dynamics. But if one was to use the same model to render a year's or century's worth of storms, the model would quickly resemble a cartoon. One would need to be concerned with the geology of the region, the vegetation on the landscape, and any man-made structures in the channel. In essence, a simple stream model can aid in rendering a simple process, but more is needed to understand the evolution of the stream's hydrological and geological system.

Coupled dual- or multi-system DGM's can be useful in understanding a single DGM's evolutionary context, and can lead to understanding of whole systems, rather than simple processes. This is not to suggest that all processes and components should be incorporated in a 
model; this leads to confusion and obfuscation, as explained later. Rather, there are some sets of problems that can benefit from coupling DGM's together.

One set of problems which has been developing rapidly is the linking of human and natural system modeling. In these instances, a human-driven system is linked to a natural process-driven system and the potential conflicts and resolutions are used o drive decisionmaking processes. This work will use the example of Land-Use Change, in the form of urbanization, and wildfire propagation, as an example of a human-natural system model. First, the complexities of linking dynamic models will be explored.

\section{Approaches to coupled dynamic spatial modeling}

The significant problems of coupling DGM's can be laid out as twofold; the technical linking of two separate models, and the semantic logic of the linking of the models (Abel et al 1994). As it becomes easier to couple models through common GIS packages, these are increasingly likely; the easier it is for a modeler to link two software process models, the less concern they may have about what exactly they are doing, and the easier it is to link two models in an incorrect way. The worst-case scenario is in a modeler linking two models, while the common elements of each model are incompatible. An often overlooked error is in an incorrect matching of spatial or temporal resolution. Generalizing a model to fit another's native resolution makes assumptions that the outputs and the model are scalable, when in fact different processes may be at work at different spatial and temporal scales. For example, a fine grain wind model at 1 meter resolution and 1 second timesteps cannot be simply generalized to a $1 \mathrm{~km}$ grid cell at 1 day intervals, or vice versa. One potential solution to this problem is using Cellular Automata (CA) as components of spatial models, as they are standardized in their spatial grain, and their temporal resolution can 
be made flexible, depending on the differences of the temporal patterns of each of the models (Park and Wagner 1997).

Coupling models occurs on a continuum from "isolated" to "loose" to "tight" to "integrated" (Nyerges 1992). An isolated model coupling consists of a hands-on manual translation of information from one model to the other. Data often need to be "massaged" in a GIS or via simple transformation or conflation to render one model's outputs into the other's inputs. A loosely coupled model still involves a user's data interaction in the data sharing, however with little or no translation of the data. A tightly coupled model uses a "Master Component" to coordinate the communication of data and parameters between the models, through a series of macros. An integrated coupled model may swap data through common interfaces, without the user's assistance. An integrated coupled model is viewed as one model, in one programming language, with no data passing; all information is stored internally. The advantage of fully integrated models is that they can run faster and more efficiently than looser coupled models. The challenge is that fully integrating two models necessitates an understanding of each model and the processes they represent, as well as intimate knowledge of the desired programming language. Yet the practical benefits of tightly coupled integrated modeling will improve along with computation speed, cross-platform emulators and the flexibility of software packages, like IDRISI, ARC/INFO and GRASS-GIS, for example.

\section{Wildfire-Landuse Coupled modeling}

Wildfire is an expensive, devastating, and persistent problem in the United States, especially as urbanization and residential development encroaches on fire adapted natural systems. On average, over the past decade, the US has spent about a billion dollars per year in wildfire 
suppression costs to fight the thousands of wildfire each year, a majority of which are triggered or initiated by humans (www.nifc.gov, accessed 12-1-06). Concurrently, the pressure of cities to expand has pushed new development into regions previously managed as wildlands. Both entities, wildfires and urban growth, have been the subject of computer models for decades, originating as aspatial spreadsheet-style models of demography or empirical wildfire spread, and evolving into sophisticated DGM's rendering urban growth and landuse change and wildfire regimes, wildfire risk and fire behavior into GIS-ready tools for policy and scientific analyses.

To create a coupled model that could incorporate the individual internal and external feedbacks of both natural and fire-adapted natural systems, the key aspects of increased wildfire risk in developing communities was identified. The coupled model had to incorporate policy changes to urbanization and wildland/wildfire management while allowing feedbacks between the two systems to be increased or decreased. To this end, the SLEUTH model of Urban Growth was chosen to be linked to a wildfire risk model.

The SLEUTH urban growth model uses a modified CA to model the spread of landuse change across an urban landscape (Clarke et al 1998). Its name comes from the GIS data layers that are incorporated into the model; Slope, Landuse, Exclusion (where growth is prohibitive, like a lake), Urban, Transportation, and Hillshade (for visualization). In a typical SLEUTH run, a region is calibrated with historical urban data, and then run into the future to produce a probabilistic map of urban propagation. While numerous wildfire spread and regime models have been created for aiding forest managers in their policy making (Li et al 1997), these models do not apply to the Wildland-urban interface (WUI), where the driving factor of wildfires is anthropogenic, and structures burn as well as wildland fuels. The Vesta model was created as a method to link the SLEUTH model to a Wildfire Risk Model that incorporated the urban fabric, 
as well as the feedbacks of a fire-adapted natural system, and policy options for potential stakeholders.

\section{The Vesta Model of Coupled Urban Growth and Wildfire Risk}

The Vesta model of Urban Growth and Wildfire Risk takes the approach that coupled models need to be linked in a way where each system can be fairly represented, and their interactions have meanings in the other system. As a result, Vesta is a model of dynamic wildfire risk, and is not a wildfire spread model. Cities and urban managers, make decisions based on short-horizon events, like large wildfires and floods, but also keep in mind a memory, albeit a fallible memory, of the historical legacy from natural disasters. Creating a model of a single fire's spread that incorporates the urban setting would not represent the natural environment in an appropriate manner for long-term urban planning. Modeling wildfire regimes is a better idea, but in Southern California (and similar climates), fire return intervals and fuel age do not represent the occurrence or intensity of wildfires, as human -caused ignition is the major cause of wildfires, often occurring during extreme weather events (Moritz et al 2004). Viewing the occurrence of wildfires along a continuum of risk, that is, the possibility of loss of property or vegetation due to fire couches the natural environment in a domain that can be planned for, and mitigated against.

Vesta is comprised of two components, SLEUTH, and the Wildfire Risk Component (WRC) (for more details on the Vesta model, refer to Goldstein 2005). In each year, the WRC generates risk by accounting for the probability of a pixel burning by the formula: Risk or $\pi=$ $\mu \rho$, where $\mu$ (or $P_{-}$ig) is the probability of a fire occurring in the region of a pixel, and $\rho$ ( or P_burn) is the probability of a pixel igniting should such a fire occur (Fried et al 1999). In this 
model, $\rho$ is a function of the fuel content of the pixel, be it a road (zero), a building (medium), or vegetation (very high). The probability of a pixel encountering a wildfire, $\mu$ is a distance-decay function from highly-ignitable regions.

\section{INSERT FIGURE 1 HERE}

One temporal iteration of Vesta operates as follows (Figure 1). First, SLEUTH is initialized with data for an urban area, in this case Santa Barbara. Next, the WRC is run and a predicted model of wildfires is calculated. The subset of the pixels that have a high probability to burn will be "burnt" and, given the predominant slope and modeled wind direction, will "burn" its surrounding pixels within a probability threshold. The newly burned urban areas will be removed from SLEUTH's Urban layer and added to SLEUTH's Exclusion layer and then SLEUTH will be run for one year. This will happen iteratively, over a specified number of timesteps, and a large number of Monte Carlo iterations. Three alternative modes of Vesta are available. The first, Urban Impacts (UI) removes the urban pixels that "Burned". The second, Urban and Exclusion Adaptation (UEA) removes "Burned" urban pixels, and adds any "Burned" pixels to the Excluded layer. The third mode, No Adaptation (NA) does neither, and essentially runs SLEUTH in parallel with the WRC, never interacting. The new (if desired) urban and Excluded layer are then passed to SLEUTH, which runs for one annual timestep. Each annual SLEUTH run has its own exclusion layer and unique urban start layer. Vesta is then run for a specified number of timesteps (years) and repeatable over any number of Monte Carlo iterations.

\section{INSERT FIGURE 2 HERE}

The Santa Barbara region was chosen due to its high urbanization pressure, its history of large wildfires in the chaparral-laden Santa Ynez Mountains that abut the region, and the rich data availability (Goldstein 2005) (Figure 2). The three modes of the Vesta Santa Barbara 
simulations were run from the year 2001 to the year 2030 for 25 Monte Carlo iterations. The results are summarized below.

\section{INSERT FIGURE 3 HERE}

\section{Model Results}

Discerning the differences between the results of all three modes of Vesta for Santa Barbara through visual inspection is challenging (see Figure 3), as the characteristics of each mode is subtle and small. Additionally, summarizing 25 Monte Carlo runs can "wash out" or generalize the fine details of each run. However, some general observations can be made. Most new urban development was forecasted for the low-lying mountain regions, which was no surprise. The UEA mode of Vesta forecasted slightly less urbanization than the other two modes, as the urbanization pressure was removed from the high-risk regions, and focused on the available agricultural lands. There were fewer forecasted fires in the UEA mode, but the UI and NA modes behaved similarly, with respect to the number of wildfires (Table 1). The UI mode forecasted larger wildfires, when they happened, when, in theory, the opposite was expected from the model.

\section{INSERT TABLE 1 HERE}

\section{Conclusions}

This paper demonstrated the need for coupled Dynamic Geospatial Models, using the Vesta model of Urban Growth and Wildfire Risk as an illustrative example. The Vesta model was demonstrated on the Santa Barbara County South Coast region, which was chosen for need, data availability, and receptive local stakeholders. However, using Santa Barbara for Vesta modeling may not have been the best choice due to its built-up nature, as most developable land has been built on, and all growth is constrained between the Pacific Ocean to the South and the mountains to the North. Vesta may find better results and utility in a less-constrained growing 
regions, of which there are many in the Western US. Vesta represents a linked model of natural processes and human decision / policy making. Even if the results are not convincing or selfevident, it can be used as a discussion starter between different groups of decision makers to see a single conflict as having impact in multiple systems, and encouraging an open decision-making process.

Once the technical and ontological obstacles are identified, understood, and rectified, coupling DGM's can lead to insights about each system, both in terms of the internal logical and nature of each model, but to a broader perspective on a single DGM's context in both a natural and social landscape. DGM's do not predict reality; Rather they are tools that can allow users and stakeholders to understand key components about the systems and processes they aim to explore and ideally understand the world at a faster and deeper level.

\section{Acknowledgements}

This work was performed under the auspices of the U. S. Department of Energy by the University of California, Lawrence Livermore National Laboratory under Contract No. W-7405Eng-48, UCRL-JRNL-222380. Additional support for this work was provided by the University of California, Berkeley with support from the Department of Homeland Security, Federal Emergency Management Agency, Assistance to Firefighters Grant Program.

\section{References}

Abel, D. J., P. J. Kilby, and J. R. Davis. 1994. The Systems Integration Problem. International Journal of Geographical Information Systems 8 (1):1-12.

Clarke, K.C. and Gaydos, L.J. 1998. Loose-Coupling a Cellular Automaton Model and GIS Long-Term Urban Growth Prediction for San Francisco and Washington/Baltimore. International Journal of Geographical Information Science. 12(7): 699-714

Fried, J. S., G. J. Winter, and J. K. Gilless. 1999. Assessing the benefits of reducing fire risk in the wildland-urban interface: A contingent valuation approach. International Journal of Wildland Fire 9 (1):9-20. 
Goldstein, N.C. 2005. Computational Testing of a Cellular Automata Urban Growth Model [dissertation]. Santa Barbara (CA): University of California; 260.

Li, C., M. Termikaelian, and A. Perera. 1997. Temporal fire disturbance patterns on a forest landscape. Ecological Modelling 99 (2-3):137-150.

Moritz, M. A., J. E. Keeley, E. A. Johnson, and A. A. Schaffner. 2004. Testing a basic assumption of shrubland fire management: how important is fuel age? Frontiers in Ecology \& the Environment 2 (2):67-72.

Nyerges, T.L. 1992. Coupling GIS and Spatial Analytic Models. In: Bresnahan PJ, Corwin E and Cowen DJ, eds. Proceedings: 5th International Symposium on Spatial Data Handling: Aug. 3-7, 1992, Charleston, S.C., USA. Columbia SC: University of South Carolina, 534-543

Park, S., and D. F. Wagner. 1997. Incorporating cellular automata simulators as analytical engines in GIS. Transactions in GIS 2 (3):213-231. 
Figures

\section{Data Flow in Vesta}

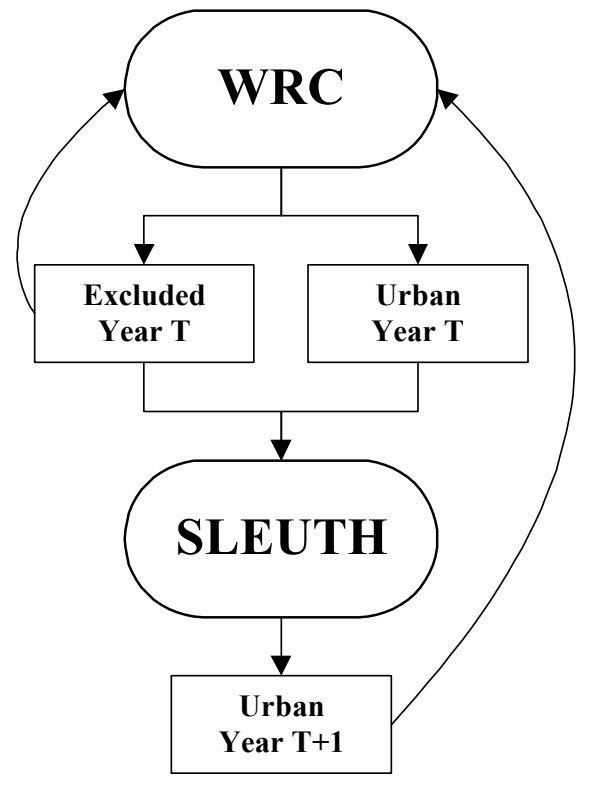

Figure 1. Flow of data from the Wildfire Risk Component to SLEUTH. 


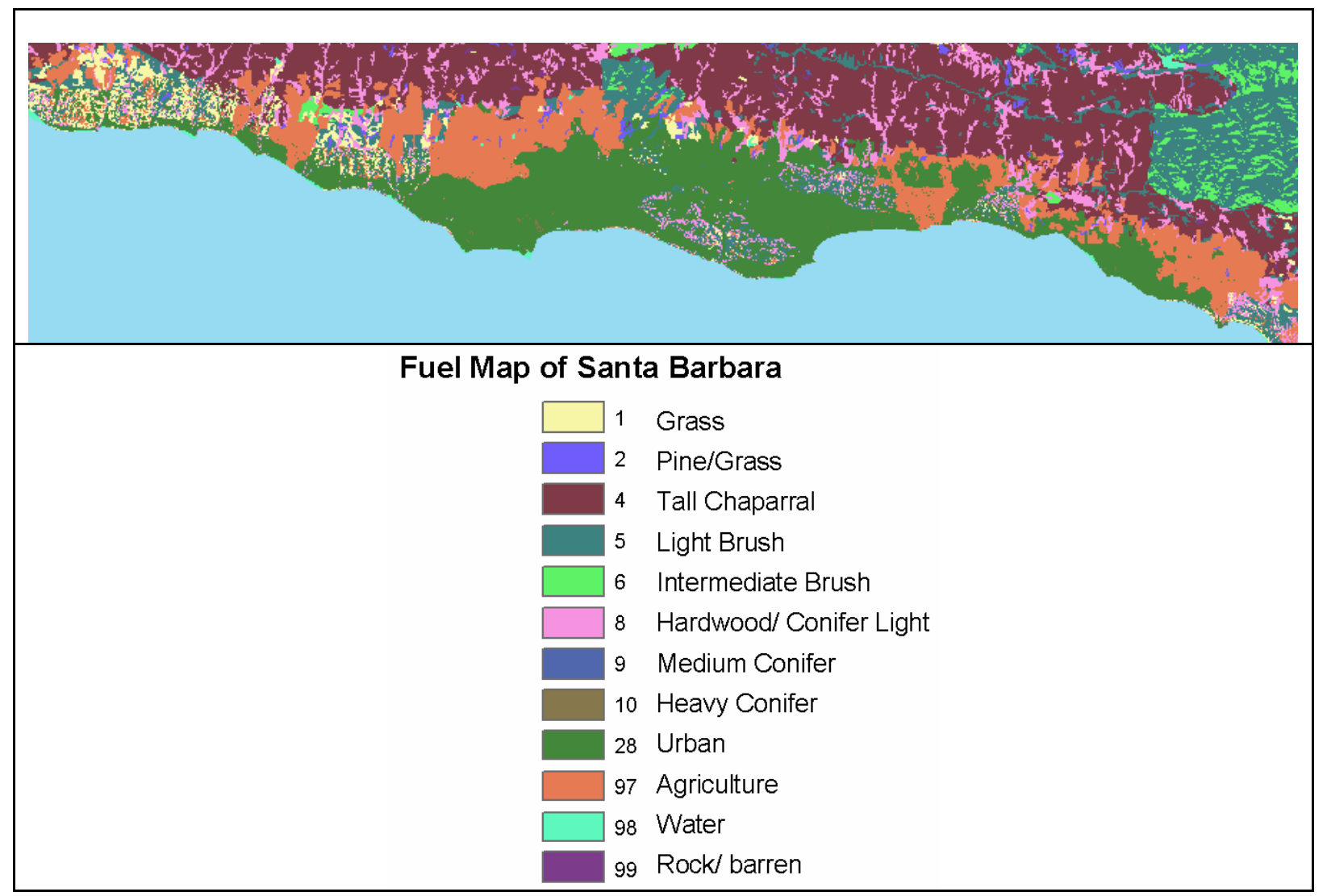

Figure 2 Fuel map of the Santa Barbara South Coast region. Data Source: California Department of Fire (frap.cdf.ca.gov) 


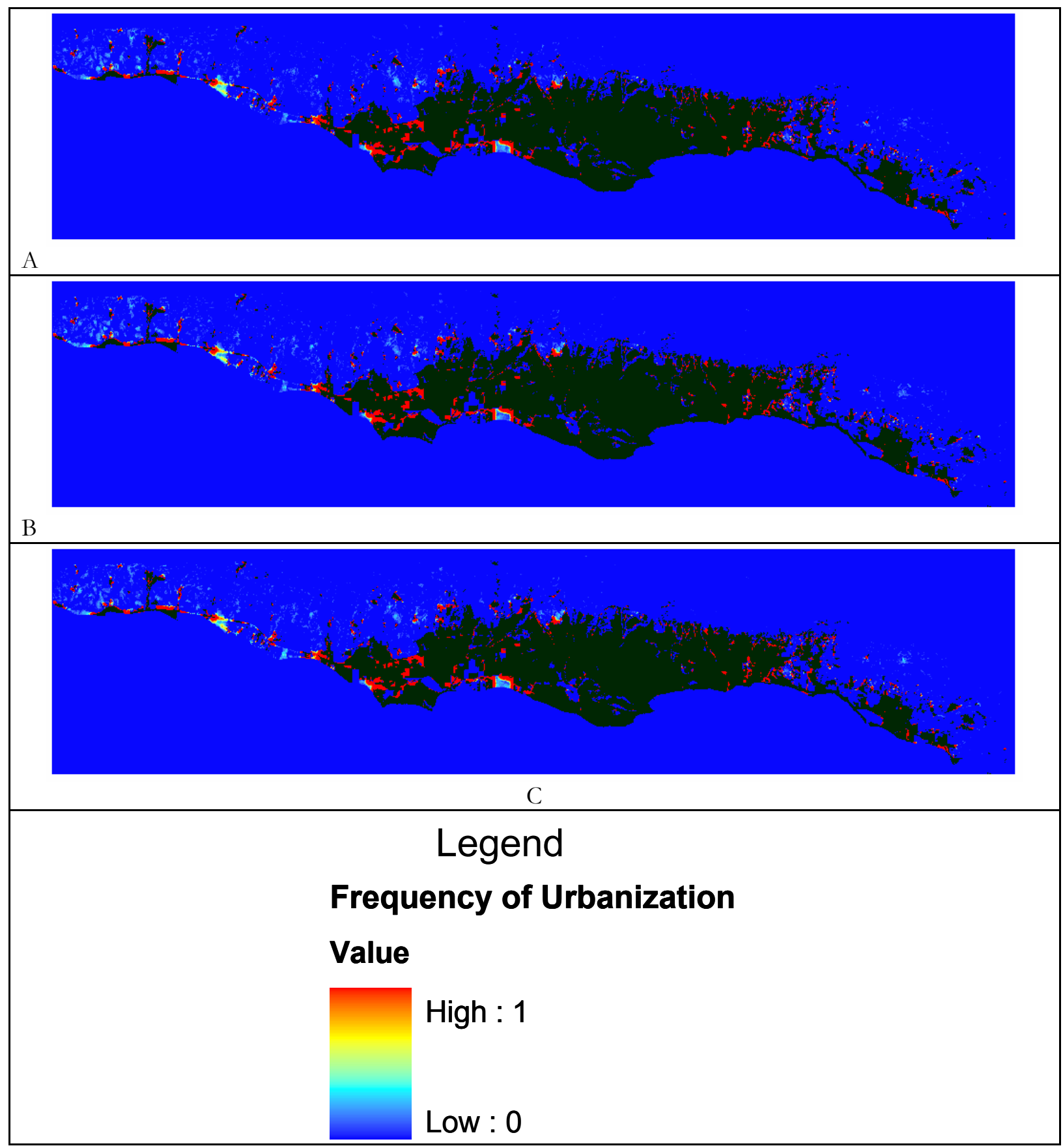

Figure 3. Map of the Vesta Model output for the "No Growth" scenario for Santa Barbara after 25 Monte Carlo iterations, for the year 2020. A. NA mode B. UI mode. C. UEA mode. The black regions are the seeded urban extent of the year 2000. The Blue regions represent area of little or no urbanization (like the ocean and high mountains). The red regions represent area that have a high probability of urbanization. Most of the differences between the runs are in the low probability regions in the foothills of the Santa Ynez Mountains. 


\begin{tabular}{|c|c|c|c|}
\hline & \multicolumn{3}{|c|}{ Mode of Vesta } \\
\hline & $\begin{array}{c}\text { No } \\
\text { Adaptation }\end{array}$ & $\begin{array}{c}\text { Urban } \\
\text { Impact }\end{array}$ & $\begin{array}{c}\text { Urban and Exclusion } \\
\text { Adaptation }\end{array}$ \\
\hline $\begin{array}{c}\text { Average final new urban growth (and } \\
\text { standard deviation) in hectares }\end{array}$ & $\begin{array}{c}1429.47 \\
29.07\end{array}$ & $\begin{array}{c}1430.19 \\
25.74\end{array}$ & $\begin{array}{c}1425.24 \\
22.41\end{array}$ \\
\hline Average number of fires & 3.09 & 3.19 & 2.92 \\
\hline $\begin{array}{c}\text { Average total burned urban size for non- } \\
\text { zero burn years }\end{array}$ & 40.32 & 44 & 39.52 \\
\hline
\end{tabular}

Table 1 - Summary table of the three modes of Vesta model runs for the No Growth Scenario of the Santa Barbara South Coast region. 